\title{
ULTRASENSITIVE VERTICAL FORCE PROBE FOR MAGNETIC RESONANCE FORCE MICROSCOPY
}

\author{
Timothy Stowe, Kevin Yasumura, Thomas Kenny \\ Departments of Applied Physics and Mechanical Engineering, \\ Terman 551, Stanford University, California, 94305-4021 \\ David Botkin, Koichi Wago, Daniel Rugar \\ IBM Research Division, Almaden Research Center, \\ San Jose, California, 95120-6099
}

\begin{abstract}
This paper reports on ultrathin single crystal silicon cantilevers with integrated in-plane tips for use in magnetic resonance force microscopy. Single crystal silicon cantilevers $400-1700 \AA$ thick and $100-150 \mu \mathrm{m}$ long have been fabricated from $\langle 100\rangle$ oriented silicon-on-insulator wafers. These cantilevers are capable of detecting forces smaller than $10^{-16} \mathrm{~N}$ in a one hertz bandwidth and have $Q$ values higher than 10,000 at pressures below $10^{-5}$ Torr. In addition to these extremely thin flexural mode cantilevers, $900 \AA$ thick silicon nitride doubletorsional mode oscillators have been fabricated as an alternative route to achieving high $Q$ resonator performance.
\end{abstract}

\section{INTRODUCTION}

Atomic force microscope techniques have been adapted for the detection of a wide variety of surface phenomena [1-2]. Recently, a new technique known as magnetic resonance force microscopy (MRFM) [3] has been established as a viable method for performing three-dimensional magnetic resonance imaging with micrometer-scale spatial resolution. Both electron spin resonance (ESR) and nuclear magnetic resonance (NMR) have been demonstrated [48]. By improving the micromachined cantilevers used for MRFM, we hope to extend the sensitivity and the spatial resolution to detect and image single electron spins with nanometer-scale spatial resolution.

Figure 1 illustrates a future experiment for the detection of individual electron spins. The experimental setup shown is housed on a vibration isolation stage inside a vacuum can placed within a liquid helium dewar. Electron spins inside the sample are excited by a frequency modulated gigahertz magnetic field originating from a coil or microwave resonator. The frequency modulation periodically flips the direction of electron spins in resonance through a process known as adiabatic inversion [5]. Since the tip of the cantilever is coated with a thin ferromagnetic film there is a magnetic dipole-dipole interaction between the tip and electron spins. The oscillating spin direction excites the cantilever though this interaction force and the resulting motion is detected using a fiber interferometer aligned onto the cantilever paddle [9].

The interaction force which drives the cantilever is given by

$$
F_{x}=\mu_{z} \frac{\partial B_{z}}{\partial x}
$$

where $\mu_{z}$ is the magnetic moment of the electron spin and $\partial B_{z} / \partial x$ is the magnetic field gradient from the ferromagnetic

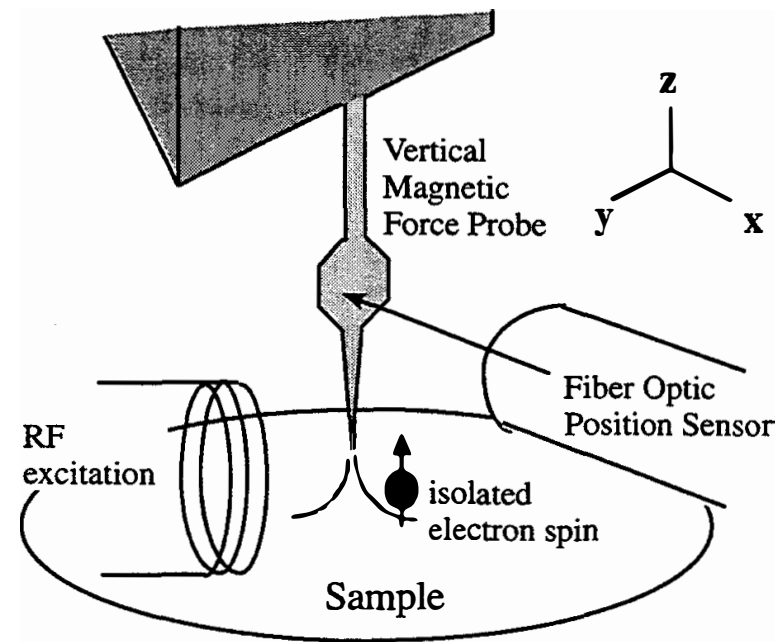

Figure 1: Experimental magnetic resonance force microscope setup.

tip. For a NiFe tip with a $500 \AA$ radius of curvature placed 150 $\AA$ above the sample surface, the interaction force is on the order of $10^{-16} \mathrm{~N}$ for a single electron. As described below, detecting such a minute force requires an extremely soft and high $Q$ cantilever. Such a cantilever operated in the standard AFM orientation, parallel to the sample substrate, would be highly susceptible to "snap down" since van der Waals forces can be as high as $10^{-9} \mathrm{~N}$. However, if the cantilever is oriented perpendicular to the sample as shown in Figure 1 the snap down problem is reduced because the cantilever's stiff axis is then normal to the substrate [10]. In order to implement this geometry, it has been necessary to develop an in-plane tip with a radius of curvature under $500 \AA$. In addition, the magnetic moment at the tip needs to point along the length of the cantilever. This requirement can be met by using tips with a narrow apex angle because this provides favorable shape anisotropy for producing magnetization parallel to the length of the cantilever.

The force resolution is the most difficult requirement imposed by this experiment. The force resolution of a micromachined cantilever in non-contact mode is limited by thermo-mechanical noise [11]. In thermal equilibrium, the thermo-mechanical displacement for a single cantilever mode is related to temperature by the equipartition theorem

$$
\frac{1}{2} k(\Delta x)^{2}=\frac{1}{2} k_{B} T,
$$


Table I, Force resolution comparison of cantilevers at $10 \mathrm{~K}$

\begin{tabular}{|l|c|c|c|c|c|c|c|}
\hline $\begin{array}{l}\text { Cantilever } \\
\text { Description }\end{array}$ & $\begin{array}{c}\text { Length } \\
(\mu \mathrm{m})\end{array}$ & $\begin{array}{c}\text { Width } \\
(\mu \mathrm{m})\end{array}$ & Thickness & $\begin{array}{c}\text { Frequency } \\
(\mathrm{kHz})\end{array}$ & Q factor & $\begin{array}{c}\text { Spring constant } \\
\left(10^{-3} \mathrm{~N} / \mathrm{m}\right)\end{array}$ & $\begin{array}{c}\text { Force Resolution } \\
\left(10^{-17} \mathrm{~N} / \sqrt{\mathrm{Hz}}\right)\end{array}$ \\
\hline thick silicon ${ }^{[13]}$ & 450 & 45 & $1.5 \mu \mathrm{m}$ & 9.8 & 200,000 & 70 & 4.3 \\
\hline silicon nitride & 60 & 10 & $800 \AA$ & 30 & 16,000 & 0.8 & 1.2 \\
\hline target cantilever & 110 & 5 & $500 \AA$ & 5.2 & 50,000 & 0.022 & 0.26 \\
\hline
\end{tabular}

where $k$ is the spring constant, $k_{B}$ is Boltzmann's constant, and $T$ is temperature. At $5 \mathrm{~K}$, a cantilever with a spring constant of $10^{-3} \mathrm{~N} / \mathrm{m}$ has thermo-mechanical displacement noise of $3 \AA$. By comparison, an interferometer can measure displacements smaller than $0.01 \AA \sqrt{ } \mathrm{Hz}$ [9]. Therefore, the thermo-mechanical noise, and not the measurement device, limits the minimum force that can be resolved from the cantilever's motion.

Taking into account the spectral distribution of the noise, the minimum detectable force in a bandwidth $\Delta B$ is

$$
F_{\text {min }}=\sqrt{\frac{2 k k_{B} T \Delta B}{\pi Q f_{o}}},
$$

where $Q$ is the quality factor and $f_{o}$ is the resonance frequency. Not all of the parameters in equation 3 can be optimized independently in order to improve the force resolution. Our MRFM experiments require that the cantilever resonance frequency fall between $5-15 \mathrm{kHz}$. The low frequency limit was chosen to reduce the influence of environmental noise while the high frequency limit was chosen to optimize the magnetic resonance signal lifetimes during cyclic adiabatic inversion [13] This finite signal lifetime, on the order of 0.1-1 seconds, limits the minimum bandwidth we can use for a single measurement. Given the constraints on the resonance frequency, the force resolution can be improved by minimizing the ratio of $k Q$ for a given cantilever geometry. For a cantilever thickness $t$, and width $w$, the length can be chosen such that the resonance frequency is fixed at $10 \mathrm{kHz}$. The dependence of the minimum detectable force on the cantilever geometry and $Q$ can then be expressed as

$$
F_{\min } \propto \sqrt{\frac{w t^{3 / 2}}{Q}}
$$

Clearly, the force resolution can be improved by reducing the cantilever thickness and width while maintaining high $Q$.

In this work we address the issue of making very thin cantilevers that maintain high $Q$ values. Previous efforts [12] have shown that it is difficult to fabricate extremely thin cantilevers ( $t \leq 2000 \AA$ ) that have high $Q$ values. One approach to obtaining a high $Q$ is to use single crystal silicon rather than an amorphous material such as silicon nitride. Another benefit of using single crystal silicon is that internal stress gradients, that can arise in amorphous materials such as silicon nitride, are a concern when performing low temperature experiments. These stress gradients can cause curvature in a cantilever as it is cooled down to liquid helium temperatures, thereby causing an undesirable reduction in the fiber optic signal.

Previous measurements by Hoen et al. [12] on silicon nitride cantilevers below $600 \AA$ thick have demonstrated $Q$ values below 3,000 at room temperature and at $10 \mathrm{~K}$. More recently we have measured $Q$ values up to 16,000 for an $800 \AA$ thick silicon nitride cantilever at $10 \mathrm{~K}$ In contrast, we have repeatedly measured $Q$ values above 50,000 on commercially available $1.5 \mu \mathrm{m}$ thick single crystal silicon cantilevers at room temperature similar to those values reported for polysilicon [14 15]. At temperatures below $10 \mathrm{~K}$, the $Q$ of these single crystal silicon cantilevers climbs to 200,000 [13]. Despite this high $Q$, the actual force sensitivity of these cantilevers is limited by their relatively high stiffness.

The first two rows in Table I show a comparison of force resolution for these cantilevers and illustrate the tradeoffs between cantilever $Q$ and cantilever stiffness. The third row represents cantilever performance that we wish to eventually achieve. Given the need for thin, high $Q$, low stress cantilevers we have investigated using bonded silicon on insulator (SOI) wafers for fabricating submicron thick single crystal silicon cantilevers.

An additional route to improving the $Q$ performance of thin oscillators is to modify their geometry. Previous work has shown that double torsional oscillators have extremely high $Q$ values. In this geometry clamping losses to the substrate are reduced [16-18]. We have modified the basic geometry of a double-torsional oscillator so that it can be used as a force probe. Because of the difficulty involved in fabricating extremely thin silicon structures, we investigated the $Q$ performance of a $900 \AA$ thick double-torsional silicon nitride oscillator and compared its performance with silicon nitride cantilever beams having similar frequencies and spring constants.

\section{SILICON CANTILEVER PROBES}

The cantilever processing begins with $<100\rangle$ oriented SOI wafers having a top silicon layer thickness of 2000-3000 $\AA$. Fabrication proceeds according to the processing sequence outlined in Figure 2. The top silicon layer is thinned down using a wet oxidation (Figure 2a). This top oxide is then removed in a buffered oxide etch (BOE) and the silicon layer is patterned using an $\mathrm{SF}_{6}$ plasma etch (Figure $2 \mathrm{~b}$ ). The details of how lateral tips are formed are described in the next section. Once the cantilevers have been patterned, polyimide is spun on the top side to protect them against scratching and as a final dry release layer. Openings in the backside oxide are patterned and the exposed silicon is etched anisotropically in $20 \%$ tetramethylammonium hydroxide (TMAH) (Figure 2c). TMAH was used rather than $\mathrm{KOH}$ because of its higher selectivity against etching the oxide mask and buried oxide layer which serves as a backside etch stop. The final release consists of removing the buried backside oxide in $\mathrm{BOE}$ and the remaining polyimide layer in an oxygen plasma (Figure 2d). Various cantilevers ranging in thickness from $400 \AA$ to $1700 \AA$ and up to 200 microns long were made without any noticeable curvature. 
a) Wet oxidation

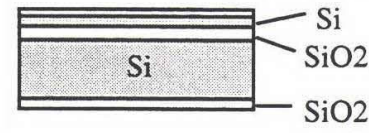

b) Pattern cantilevers

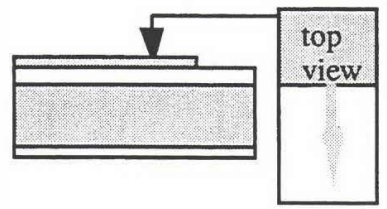

c) Etch back

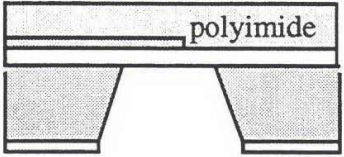

d) Dry release

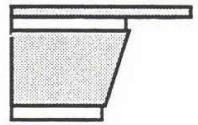

e) Evaporate $\mathrm{NiFe}$

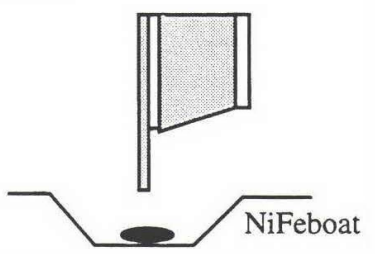

Figure 2: Processing Sequence

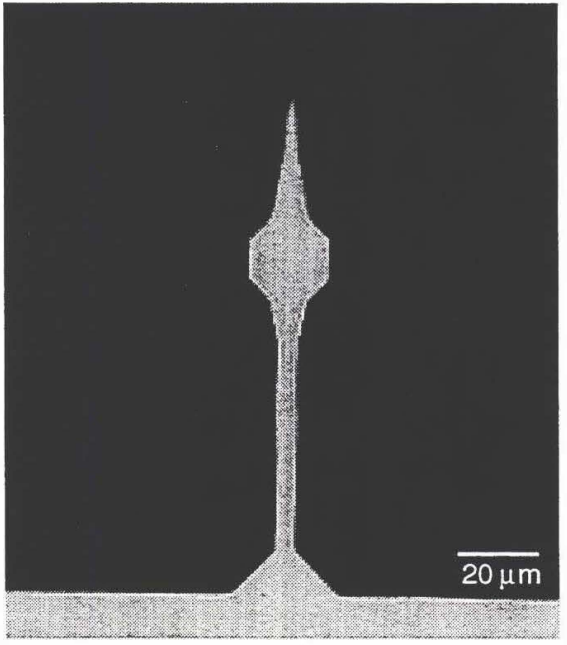

Figure 3: Finished "Spearhead" cantilever $120 \mu \mathrm{m}$ long and $0.17 \mu \mathrm{m}$ thick.

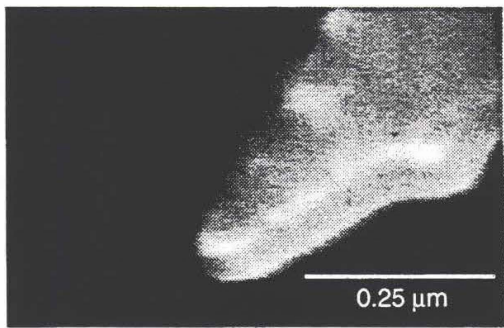

Figure 4: A cantilever tip formed by lateral oxidation sharpening.
In addition to the dry oxygen plasma release process, we have successfully experimented with $\mathrm{BOE}$ wet release processes without the top polyimide layer. In this case, the final release step consists of critical point drying [19] to avoid meniscus forces which lead to surface adhesion of the cantilevers.

\section{FABRICATION OF IN-PLANE TIPS}

Three possible methods were considered for lateral tip formation: lateral oxidation sharpening, convex comer undercutting, and edge intersection from a double patterning procedure (Figure 5). The lateral oxide sharpening method is similar to that used by Yao and MacDonald [20-21] for out of plane tips. First a layer of silicon nitride greater than $1000 \AA$ thick is grown over the silicon and the cantilever geometry is

a) Lateral oxidation sharpening

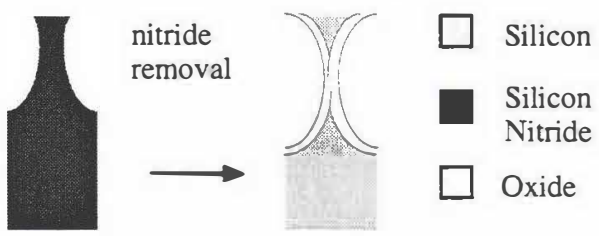

b) Convex comer undercutting

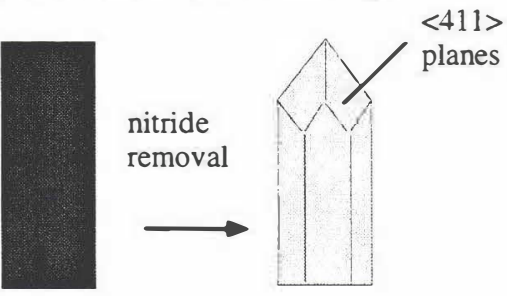

c) Mask offset patteming

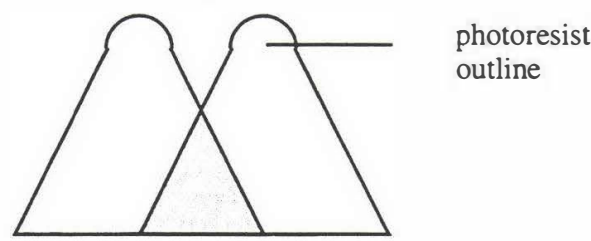

(blow up of the tip region)

Figure 5: Three Lateral Tip Processes

patterned with a tip geometry shown in Figure 5a. The silicon nitride layer serves as an oxidation mask. Next a $900^{\circ} \mathrm{C}$ oxidation is used to laterally oxidize the underlying silicon, pinching off the silicon to form a tip. Finally, the nitride layer is removed in phosphoric acid. This tip sharpening process has yielded tip radii under $500 \AA$ (Figure $3 b$ ) for tip apex angles of approximately 30 degrees. We found that smaller tip apex angles sometimes cause tearing in the top silicon nitride mask due to stress between the silicon nitride and oxide layers.

A second possible method for forming lateral tips uses an anisotropic etch to define the tip. Silicon nitride is grown over the silicon cantilevers and patterned so that it protects all areas of the cantilever except for the end, leaving two exposed convex comers. An anisotropic etch such as $\mathrm{KOH}$ will undercut 
these exposed convex comers, forming a tip at two intersecting $<411>$ planes in the silicon [ 2 ]. Although this method may be very suitable for other applications requiring in-plane tips we did not investigate this method further because the tip apex angle between the intersecting $\langle 411\rangle$ planes is limited to 62 degrees which, as mentioned earlier, is not optimal with respect to magnetic shape anisotropy once a NiFe film is evaporated.

Finally, a very successful technique we have explored involves both a double exposure and double etch to define a single in-plane tip [23] (Figure 5c). First a $1.0 \mu \mathrm{m}$ layer of photoresist defines the cantilever with a $0.5 \mu \mathrm{m}$ rounded tip. The cantilever is patterned as usual in an $\mathrm{SF}_{6}$ etch. The first photoresist mask is stripped and a second photoresist mask containing the same pattern is applied again but shifted laterally by $1 \mu \mathrm{m}$. The second dry etch forms a tip at the intersection of the sidewall formed during the first etch. This process can be used to form tips with radii below $500 \AA$ and with high aspect ratios. In addition, these tips can be fabricated using many different materials including silicon nitride. The vertical sharpness of the tip is limited only by the thickness of the material. We have found this process to require significantly shorter processing times and has improved tip-to-tip uniformity.

Evaporation was used to place ferromagnetic material on the end of the tip. However because these cantilevers are extremely thin, simple evaporation onto one side of the tip can cause dramatic bending of the tip even for film thickness below $350 \AA$ over tip lengths of only a few $\mu \mathrm{m}$. This is caused by the tensile stress of the film that develops during deposition. Therefore, in order to place magnetic material such as $\mathrm{Ni}, \mathrm{Co}$, or $\mathrm{NiFe}$ at the end of a sharp tip, we have developed an edge-on evaporation technique shown in Figure 2e. Edge-on evaporation maximizes the amount of material deposited onto the very end of the tip and reduces material deposited along the length of the cantilever. Furthermore, edge-on evaporation balances the amount of material deposited on both sides of the tip. During evaporation, the side that receives the greater flux of material will tend to bend away from the source, increasing the deposition rate onto the opposite side. This self-correcting process causes no noticeable bending provided the cantilever is aligned perpendicular to the evaporation source before the start of the evaporation. Figure 3 shows a finished cantilever after edge-on evaporation.

\section{DOUBLE-TORSIONAL SILICON NITRIDE PROBE}

To fabricate the double-torsional probe, a layer of low stress silicon nitride $900 \AA$ thick was grown on top of a $<100>$ oriented silicon wafer and patterned as shown in Figure 6. The exposed silicon surrounding the silicon nitride oscillators was etched in a solution of 20\% TMAH until the entire silicon nitride structure was undercut leaving it supported by the $4 \mu \mathrm{m}$ wide beam running vertically through Figure 6 . The oscillators were stored in water and finally released using critical point drying. The geometry was chosen such that moments of inertial about the central supporting beam are balanced. The inner torsional element has a tip which extends past the outer oscillator in order to allow sample approach. The high $Q$ torsional mode results from counter oscillation of the outside frame and the inside probe. The fiber interferometer was aligned to the middle paddle located to the left of torsional beam support in Figure 6.

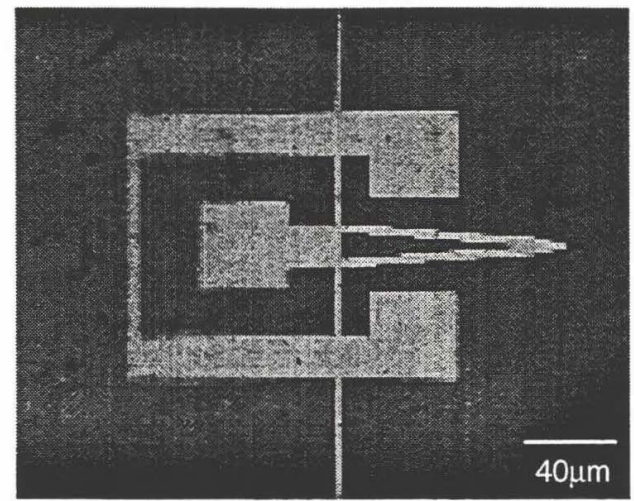

Figure 6: Optical micrograph of the double torsional force probe. The torsional beam supports the structure at both ends and is $41 \mathrm{~m}$ wide and over $200 \mu \mathrm{m}$ long.

\section{Q MEASUREMENTS}

A fiber optic interferometer was used in an evacuated bell jar to perform room temperature $Q$ measurements. $Q$ values were determined by measuring both decay times and amplitude vs. frequency curves. Figure 7 shows typical $Q$ vs. pressure results for a bare silicon cantilever (no magnetic coating) that is 0.3 $\mu \mathrm{m}$ thick with a resonance frequency of $30 \mathrm{kHz}$. The lowest pressure shown in Figure $7,2 \times 10^{-3}$ Torr, was the lowest obtainable inside our bell jar and clearly shows that the highest measured $Q$ value of 18,000 was still pressure limited.

Lower pressures (below $10^{-5}$ Torr) were obtained inside a low temperature vacuum apparatus. $Q$ vs. temperature measurements are shown in Figure 8 for a bare silicon cantilever with the same dimensions as shown in Figure 3 and a resonance frequency of $14 \mathrm{kHz}$. The $Q$ increased monotonically as temperature is decreased due to freeze-out of energy dissipation mechanisms. At $5 \mathrm{~K}$ and $10^{-6} \mathrm{Torr}, Q$ values as high as 26,000 were measured. After coating this cantilever with 350 $\AA$ of $\mathrm{NiFe}, \mathrm{a} Q$ of 22,000 was obtained at low temperature.

These $Q$ values represent the largest we have measured for a cantilever below $0.2 \mu \mathrm{m}$ in thickness, but they are still lower than the $Q$ of 200,000 we have measured for $1.5 \mu \mathrm{m}$ thick silicon cantilevers [13]. We attribute the lower $Q$ in the thinner cantilevers to the greater importance of surface losses which may arise from surface dislocations and other microscopic imperfections. One possibility for increasing the $Q$ is to do a forming gas anneal in order to reduce the surface dislocations and dangling bonds that may limit the cantilever $Q$.

In order to calculate the noise sensitivity of these soft cantilevers it is necessary to determine their spring constants. Attempting to measure load vs. displacement curves is impractical given that forces below $10^{-11} \mathrm{~N}$ would need to be applied directly at the tip. A more precise method for determining the spring constant is to directly measure the RMS thermal vibration noise. At $7 \mathrm{~K}$ the RMS thermal noise amplitude was $2 \AA$, giving a spring constant of about $2 \times 10^{-3} \mathrm{~N} / \mathrm{m}$. Neglecting variations in the beam shape from the paddle and tip, this value is within $10 \%$ of that predicted by simple beam theory.

Using the measured values for the spring constant, frequency, and $Q$, the calculated force resolution of the cantilever (shown in Figure 3) was $2 \times 10^{-17} \mathrm{~N} \sqrt{ } \mathrm{Hz}$ at $10 \mathrm{~K}$. This 
force resolution is the best we have measured and should be sufficient for single electron spin detection.

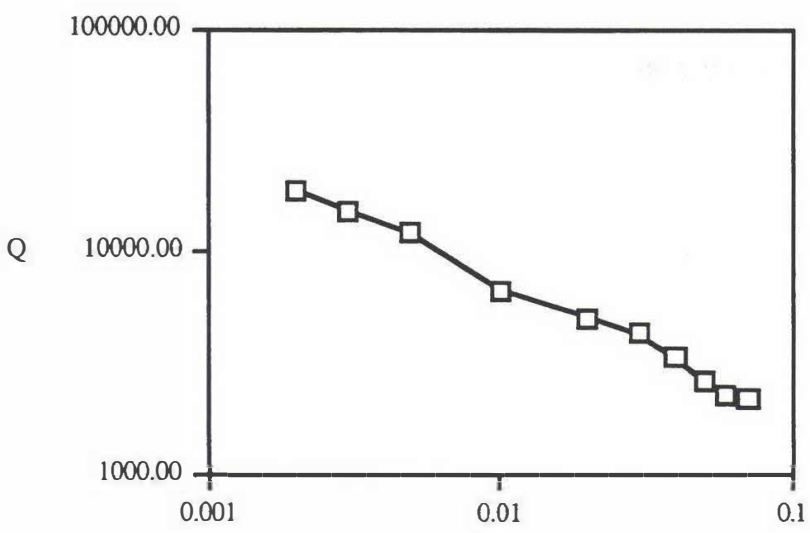

Pressure (Torr)

Figure 7: $Q$ vs. Pressure for a $0.3 \mu \mathrm{m}$ thick silicon cantilever

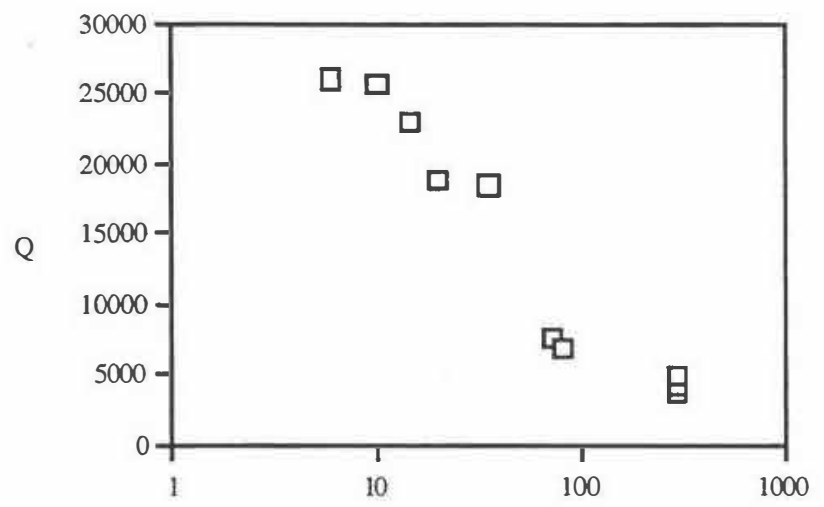

Temperature ${ }^{\circ} \mathrm{K}$

Figure 8: $Q$ vs. Temperature for a $0.17 \mu \mathrm{m}$ thick cantilever.

Lastly, the $Q$ performance of the double torsional oscillator shown in Figure 6 was investigated. At $10 \mathrm{~K}$ there were four high $Q$ modes ranging in frequency from 4.8 to 23 $\mathrm{kHz}$. The highest $Q$ modes at $4.8 \mathrm{kHz}$ and $17.5 \mathrm{kHz}$ had $Q$ values of 20,000 . This oscillator has the highest $Q$ we have measured for silicon nitride oscillators of comparable thickness. this result is $25 \%$ greater than that obtained for a simple silicon nitride cantilever beam (Table I). The mode of oscillation (flexural vs. torsional) and cantilever geometry continue to play a role in determining the $Q$ for these extremely thin oscillators However, we note that the $Q$ improvement we measured is not as pronounced as improvements reported by Buser [17] for much thicker silicon oscillators.

\section{SURFACE MEASUREMENTS}

These cantilevers must maintain their high $Q$ as the tip approaches a sample surface in order to maintain high force sensitivity. Sample approach measurements taken at room temperature inside a bell jar indicate that these cantilevers maintain relatively high $Q$ until surface interactions begin to introduce damping 100-1000 $\AA$ from the sample. Approach measurements also show an increase in the natural resonance frequency of the cantilever due to van der Waals forces. This behavior is similar to that seen by Gregor, et al. [24] in which a tapered fiber optic tip was driven at resonance over a sample surface.

In order to verify that the end of the tip was magnetic after the edge-on evaporation, these cantilevers were used to image the bit patterns on a disk drive sample. The resonance frequency of the cantilevers was sensitive to magnetic field gradients in the lateral direction shown in Figure 9. Figure 10 shows a magnetic force microscopy (MFM) image of disk drive tracks taken approximately $5000 \AA$ above the surface by measuring the phase of the cantilever oscillation while it was driven at a constant frequency of $12 \mathrm{kHz}$ to a vibration amplitude of $100 \AA$.

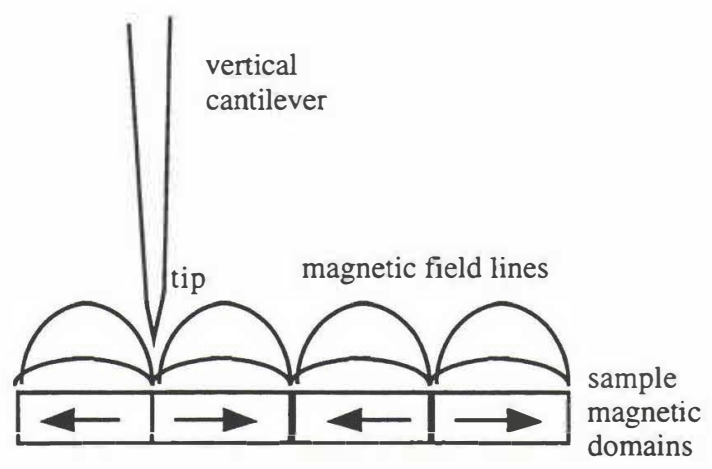

Figure 9: Illustration of the magnetic force microscopy technique.

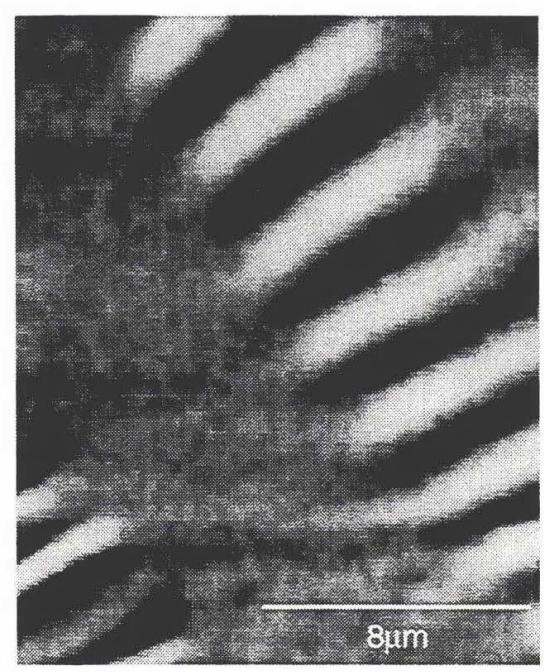

Figure 10: Imaged $8 \mu \mathrm{m}$ wide disk drive track. 


\section{CONCLUSIONS}

We have investigated both flexural and torsional mode oscillators for magnetic resonance force microscopy. In particular, we demonstrated ultrathin single crystal silicon cantilevers with sharp in-plane magnetic tips that are operated perpendicular to the substrate. Three techniques for making inplane tips have been explored yielding tip radii below $500 \AA$ and narrow tip apex angles. The predicted field gradients from these in-plane magnetic tips should produce interaction forces as high as $10^{-16} \mathrm{~N}$ for a single electron spin. In a one hertz detection bandwidth, the force resolution of these cantilevers approaches $10^{-17} \mathrm{~N}$. Thus, single electron magnetic resonance force microscopy should be feasible.

\section{ACKNOWLEDGMENTS}

We acknowledge S. Hoen's work in the design and testing of our critical point dryer and are grateful for invaluable processing advice from Chris Storment, Ron Maynard, John Suh, and Steve Minne at the Center for Integrated Systems. T. Stowe received funding for this research from the John and Fannie Hertz Foundation. D. Botkin received partial support from the Office of Naval Research. This work has also been supported by the National Science Foundation CAREER Award (ECS-9502046), The Charles Lee Powell Foundation, and the Terman Fellowship. We would also like to acknowledge the National Science Foundation for travel support for this conference.

\section{REFERENCES}

1. G. Binning, C. F. Quate, and C. Gerber, Physics Review Letters, 56, 930 (1986).

2. D. Rugar, P. Hansma, "Atomic Force Microscopy", Physics Today, 43 (1990).

3. J. A. Sidles,Phys. Rev. Lett., 58, 2854 (1991).

4. O. Züger and D. Rugar, "First images from a Magnetic Resonance Force Microscope", Applied Physics Letters, 63, 2496-2498 (1993).

5. J. A. Sidles, et al, "Magnetic Resonance Force Microscopy”, Review of Modern Physics, 67, No 1, pp. 249-265 (1995).

6. D. Rugar, C. S Yannoni, J. A. Sidles, "Mechanical Detection of Mangetic Resonance", Nature, 360, pp.563-566 (1992).

7. D. Rugar, et al., "Force Detection of Nuclear Magnetic Resonance”, Science, 264, pp.1560-1563 (1994).

8. P.C. Hammel, et al, "Sub-Surface Imaging with the Magnetic Resonance Force Microscope", Journal of Low Temperature Physics, 101, No. 1/2, pp.59-69 (1995).

9. D. Rugar, H. J. Mamin, P. Guethner, Applied Physics Letters, 55 , pg. 2588 (1989).
10. A. DiCarlo, et al, "Magnetic force microscope utilizing an ultra-small-spring-constant vertically cantilevered tip", Ultramicroscopy, 47, pp. 383-392 (1992).

11. T.B. Gabrielson, "Mechanical-thermal noise in micromachined acoustic and vibration sensors.", IEEE Trans. on Electron Devices, 40, No.5, pp. 903-909 (1993).

12. S. Hoen, et al, "Fabrication of Ultrasensitive Force Detectors", Proceedings of the Solid State Sensor and Actuator Workshop, pp. 209-212 (1994).

13. K. Wago, et al, "Low Temperature Magnetic Resonance Force Detection", J. Vac. Sci. Tech B, March/April, (in press) (1996).

14. H. Guckel, et al., "Polysilicon Resonant Microbeam Technology for High Performance Sensor Applications", Proceedings of the Solid State Sensor and Actuator Workshop, pp 153-156 (1992).

15. J. D. Zook, et al., "Characteristics of polysilicon resonant microbeams", Sensors and Actuators A,35, pp 51-59 (1992).

16. R. N. Kleiman, et al., "Single-crystal silicon high-Q torsional oscillators", Review of Scientific Instruments, 56, pp. 2088-91 (1985).

17. R.A. Buser, et al., "Resonant Silicon Structures", Sensors and Actuators, 17, pp. 145-153 (1989).

18. R. A. Buser and N. F. de Rooij, "Very high Q-factor resonators in monocrystalline silicon torsional resonators", Sensors and Actuators, A21-A23, pp. 323-327 (1990).

19. G. T. Mulhern, D. S. Soane, and R.T. Howe, 1993 International Conference on Solid-State Sensors and Actuators: Transducers '93, Yokohama, Japan (1993).

20. J. J. Yao, N. C. MacDonald, "Fabrication of high frequency two-dimensional nanoactuators for scanning probe devices", $J$. Micromechanical Systems, 1, pp. 14-22 (1992).

21. J. J. Yao and N. C. MacDonald, "A micromachined, singlecrystal silicon, tunable resonator", J. Micromechanical Microengineering, 6, pp. 257-264(1996).

22. H. Sandmaier, et al, "Comer Compensation Techniques in Anisotropic Etching of (100)-Silicon using Aqueous KOH", International Conference on Solid-State Sensors and Actuators (1991)

23. Kobayashi, D., et al., "Control of a micro tunneling unit under atomic force", IEEERSJ Intermational Conference on Intelligent Robots and Systems. Human Robot Interaction and Cooperative, 2, (1995).

24. M. J. Gregor, et al., "Probe-surface interaction in near-field optical microscopy: The nonlinear bending force mechanism", Applied Physics Letters, 68., No. 3, pp. 307-309 (1996). 